\title{
PRODUCTION AND EVALUATION OF HIGH FOLIC ACID BALADY BREAD
}

(Received: 19.11.2012)

\author{
By \\ A.E. Mohamed, H. F. Ahmed, M. A. Atwa*and N. M. Abd El-Motaleb \\ Food Technology Research Institute, *Regional Center for Food and Feed, \\ Agricultural Research Center, Giza, Egypt
}

\begin{abstract}
Deficiency of folic acid in human lead to macrocytic (megaloblastic) anemia and leukopenia, hence, this study targeted to utilization of by-product which obtained during mill wheat and characterized by its high folic acid content. Total folic acid (TFA) was determined in fine bran (FB) and wheat germ (WG) and they found to contain 316 and $286 \mu \mathrm{g} / 100 \mathrm{~g}$, respectively. Balady bread samples (prepared from wheat flour 82 and $72 \%$ extraction rate) were fortified by different levels of FB and WG. The results indicated that TFA contents increased from 124 to $318 \mu \mathrm{g} / 100 \mathrm{~g}$ in balady bread samples fortified by FB and WG. Chemical composition of balady bread samples fortified by FB and WG showed the same trend since; protein, fat, fiber and ash were increased. Values of total dietary fiber, iron, zinc and calcium were also enhanced. Sensory evaluation results showed that there were no significant differences among all balady bread samples for diameter and separation of layers, but other characteristics of sensory evaluation were adversely affected with the exception of balady bread samples prepared from wheat flour $72 \%$ extraction containing different levels of FB and WG. Accordingly, FB and WG can be recommended as natural rich sources of folic acid supplement for balady bread produced from wheat flour $72 \%$ extraction rate.
\end{abstract}

Key words: balady bread, fine bran, folic acid, wheat germ.

\section{INTRODUCTION}

Folic acid is the group name (also termed folates or folacin) given to a number of yellow crystalline compounds related to pteroglutamic acid. Folic acid is one of the water soluble B vitamins .It is found in whole wheat, soy bean, yeast, wheat germ, wheat bran and green leafy vegetable. So, these products could be considered an ideal natural supplement for producing folic acid baked products. Among cereals, wheat (approx. $363 \mathrm{~g} /$ capita/day) is frequently consumed as balady bread with each meal. In Egypt, they are the main dietary energy source, providing about $66 \%$ of energy (FAO 2005).

Folic acid deficiency during pregnancy has been found to cause neural tube defects (NTD) in newborn babies (West et al., 2012). The role of folic acid in prevention of ischaemic heart disease, prevention of leukopenia, decreasing plasma homocysteine, prevention of colorectal cancer, prevention of neuro cognitive decline and prevention of macrocytic (megaloblastic) anemia has also recently received increased attention
(Houghton et al., 2011, Kim et al., 2012 and Walker et al., 2012).

An alternative to folic acid fortification of stable foods could be to increase the folate content in cereal foods, either by bio - processing methods such as germination (e.g. cereal grains) and fermentation (e.g. bread) or by use folate rich fractions obtained by milling for maintenance of optimal health, as well as prevention of disease (Katina et al., 2007 and Hefni \& Witthöft 2011).

With the use of novel milling technology, it has become commercially viable to isolate the aleurone layer of cells from wheat grain and to prepare a novel flour from this fraction that has a natural folate concentration of; $500 \mu \mathrm{g} / 100 \mathrm{~g}$. This study has shown that products made from wheat aleurone flour is a good source of bioavailable, natural folate (Fenech et al., 1999).

Fortification of the wheat flour used for balady bread baking with iron and folic acid was recently introduced in Egypt (personal communication with Prof Azza Gohar, National Nutrition Institute, Cairo, Egypt) since these micronutrient 
deficiencies are widespread. Anaemia is a major public health problem with a prevalence of 30$50 \%$ among children aged 2-5 years and women of reproductive age (EDHS, 2005), which can be a result of iron, folate or vitamin B12 deficiency. A complement or alternative to mandatory folic acid fortification could increase the natural folate content in Egyptian balady bread by bioprocessing techniques.

Balady bread is the most popular items consumed nearly by all levels of society in Egypt, ready to eat, availability in different time and affordable cost, thereby, the present study has been planned (1) to produce balady bread with increased folic acid content (2) to produce balady bread fortified by fine bran and wheat germ (3) to evaluate different balady bread samples.

\subsection{Materials}

\section{MATERIALS AND METHODS}

Whole wheat, wheat flour (82 and $72 \%$ extraction rate), wheat fine bran and wheat germ were obtained from Middle and West Delta Mills Company, Benha City, El Qualiubiya Governorate, Egypt. Active dry yeast was purchased from local supermarket.

\subsection{Methods}

\subsubsection{Preparation of whole wheat flour and wheat germ meal}

Whole wheat sample was milled via laboratory mill (1400, Perten) to get the whole wheat flour. While wheat germ was first roasted at $135^{\circ} \mathrm{C}$ for $15 \mathrm{~min}$. then milled to obtain wheat germ meal.

\subsubsection{Preparation of balady bread}

Balady bread formulas are shown in Table (1). Balady bread prepared as the following procedures: one hundred gram of each sample was mixed with water (as formation of dough need), $1 \%$ sodium chloride and $1.5 \%$ active dry yeast. The ingredients were kneaded for 20 min. in mixer laboratory mechanical. The dough was fermented for one hr. at $30^{\circ} \mathrm{C}$ under $85 \%$ relative humidity (RH). Dough was divided into 100 gm pieces. Pieces were molded on a wooden board previously sprinkled with fine layer of bran and left for $15 \mathrm{~min}$. at $30^{\circ} \mathrm{C}$ and $85 \%(\mathrm{RH})$. The pieces were flattend to about $20 \mathrm{~cm}$ diameter and $0.5 \mathrm{~cm}$ thickness then proofed at $30^{\circ} \mathrm{C}$ and $85 \%(\mathrm{RH})$ for $30 \mathrm{~min}$. then baked at $450-500^{\circ} \mathrm{C}$ for $1-2 \mathrm{~min}$. in electric oven. Bread loaves were allowed to cool on rack for $30 \mathrm{~min}$. before organolyptic evaluation, and then packed in polyethylene bags until next analysis according to the method of Mohamed et al. (1996).

\subsubsection{Determination of chemical composition}

Moisture protein, fat, crude fiber and ash contents were determined according to the methods of AOAC (2000) and carbohydrate calculated by difference. Minerals content (iron, zinc and calcium) were digested according to the method of AACC (2000) and determined by Atomic Absorption Spectrophotometry (model 3300, Perkin-Elmer, Norwalk). Total dietary fiber was estimated according to the method of AACC (2000).

\subsubsection{Determination of total folic acid}

Reversed-phase gradient HPLC method was performed. Using the HPLC instrument. Dionex (ASI-100 automated sample injector - P680 $\times$ pump gradient - $170 \mu-\mathrm{U} . \mathrm{V}$ detector) according to the method of Póo - Prieto et al. (2006). The folates were separated on an ODS-hypersil $(5 \mu \mathrm{m}$, $4.6 \times 250 \mathrm{~mm}$ ) analytical column. A flow rate of 1 $\mathrm{ml} / \mathrm{min}$ was used. The mobile phase program consisted of $3 \mathrm{~min}$ with $100 \%$ A $(28 \mathrm{mmol} / \mathrm{L}$ diabasic potassium phosphate and $60 \mathrm{mmol} / \mathrm{L}$ phosphoric acid in water) followed by a linear gradient of $10 \mathrm{~min}$ to $70 \% \mathrm{~A}: 30 \% \mathrm{~B}(28$ $\mathrm{mmol} / \mathrm{L}$ dibasic phosphate) and $60 \mathrm{mmol} / \mathrm{L}$ phosphoric acid in $200 \mathrm{ml} / \mathrm{l}$ acetonitrile and 800 $\mathrm{ml} / \mathrm{L}$ water). A second linear gradient from $70 \%$ A: $30 \%$ B to $45 \%$ A: $55 \%$ B was then run over the next $17 \mathrm{~min}$, followed by a third linear gradient to 43\% A: 57\% B over the next $15 \mathrm{~min}$. At $45 \mathrm{~min}$, the column was equilibrated for $5 \mathrm{~min}$ in the initial conditions and another sample analysis could be initiated immediately. The absorbance of folic acid was monitored with a detector set at 280 $n$ m,using standard folic acid solution.

\subsubsection{Bread texture profile analysis:}

The changes in the texture profile of bread due to staling were measured using the penetration test. A QTS texture analyzer (CNS Farnell, Hertfordshire, UK) was used to measure the force required for penetration of a round - bottom (2.5 $\mathrm{cm}$ diameter $\times 1.8 \mathrm{~cm}$ height) probe at a velocity of $30 \mathrm{~mm} / \mathrm{min}$ into the bread. The settings of the texture analyzer were: Trigger Value at $0.05 \mathrm{~N}$ and Target Value at $30 \mathrm{~mm}$ according to the method of Sourki et al. (2010).

\subsubsection{Sensory evaluation}

The balady bread loaves were sensory evaluated for color \& appearance, diameter, separation, biting textures, aroma, taste and crumb texture according to method of Mohamed et al., (1996).

\subsubsection{Statistical analysis}

The gathered results in present study were statistically analyzed using the SPSS version 10.0 
Table (1): Preparation of different formulas.

\begin{tabular}{|c|c|c|c|}
\hline $\begin{array}{c}\text { Samples } \\
(\text { No. })\end{array}$ & $\begin{array}{c}\text { Wheat flour } \\
(\mathbf{g})\end{array}$ & $\begin{array}{c}\text { Fine bran } \\
(\mathbf{g})\end{array}$ & $\begin{array}{c}\text { Wheat germ } \\
(\mathbf{g})\end{array}$ \\
\hline 1 & Whole wheat flour $(100)$ & - & - \\
\hline 2 & $82 \%$ extraction $(100)$ & - & - \\
\hline 3 & $72 \%$ extraction $(100)$ & - & - \\
\hline 4 & $82 \%$ extraction $(75)$ & 25 & 25 \\
\hline 5 & $82 \%$ extraction $(75)$ & - & 10 \\
\hline 6 & $82 \%$ extraction $(75)$ & 15 & 15 \\
\hline 7 & $82 \%$ extraction $(75)$ & 10 & - \\
\hline 8 & $72 \%$ extraction $(75)$ & 25 & 25 \\
\hline 9 & $72 \%$ extraction $(75)$ & - & 10 \\
\hline 10 & $72 \%$ extraction $(75)$ & 15 & 15 \\
\hline 11 & $72 \%$ extraction $(75)$ & 10 & \\
\hline
\end{tabular}

windows program (SPSS, Inc., 1998, Chicago, II). Data were expressed as means \pm SEM. Statistical analysis was performed using one-way analysis of variance followed by Tukey post hoc test. Duncan's tests were done to compare a pair of group means.

\section{RESULTS AND DISCUSSION}

\subsection{Chemical composition of raw materials}

Raw materials were chemically analyzed and the results were summarized in Table (2). The data revealed that the highest protein and ash contents $\mu \mathrm{g} / 100 \mathrm{~g}$ ) was found in active dry yeast followed by fine bran $316 \mu \mathrm{g} / 100 \mathrm{~g}$, wheat germ meal 286 $\mu \mathrm{g} / 100 \mathrm{~g}$, whole wheat flour $43 \mu \mathrm{g} / 100 \mathrm{~g}$, wheat flour $82 \%$ extraction $32 \mu \mathrm{g} / 100 \mathrm{~g}$ and wheat flour $72 \%$ extraction $28 \mu \mathrm{g} / 100 \mathrm{~g}$. These results are in concide with those noticed by Reed \& Nagodawithcnan (1991), Osseyi el al., (2001), Hefni et al., (2010) and Hefni \& Witthöft (2012). On the other side, Jägerstad et al., (2005) mentioned that folates are concentrated to the outer layers of the kernel wheat and fractions taken from different parts of the kernel wheat can

Table (2): Chemical composition (\%) of raw materials (on dry weight basis)*.

\begin{tabular}{|l|c|c|c|c|c|}
\hline \multicolumn{1}{|c|}{ Samples } & Protein & Fat & Fiber & Ash & Carbohydrates $^{* *}$ \\
\hline Whole wheat flour & 15.29 & 2.13 & 3.01 & 2.22 & 77.35 \\
\hline Wheat flour (82\% ext) & 11.12 & 1.78 & 1.81 & 1.45 & 83.84 \\
\hline Wheat flour (72\% ext) & 10.23 & 1.14 & 0.61 & 0.53 & 87.49 \\
\hline Fine bran & 17.80 & 4.50 & 8.60 & 5.80 & 63.30 \\
\hline Wheat germ & 21.40 & 9.50 & 2.50 & 5.40 & 61.20 \\
\hline Active dry yeast & 44.17 & 1.23 & 1.97 & 6.43 & 46.20 \\
\hline
\end{tabular}

Each value is a mean of duplicate determination.

(44.17 and 6.43\%), respectively were found in active dry yeast. Meanwhile, wheat germ meal was rich in fat content $(9.50 \%)$. In the meantime, fine bran characterized by its higher fiber content $(8.60 \%)$ than that of other raw materials. At the same time, wheat flour (72 and $82 \%$ extraction) was higher in carbohydrates content (87.49 and $83.84 \%$ ) consecutively than that of other raw materials. These data are similar to results obtained by Reed \& Nagodawithenan (1991), Sidhu et al. (1999), Farouk et al. (2002), Louz et al., (2010) and Metwalli and Abdelrasoul (2010).

\subsection{Folic acid content of raw materials}

With respect to total folic acid (TFA) content of raw materials, the obtained data in Table (3) showed that the highest TFA content (1652
Table (3): Folic acid content $(\mu \mathrm{g} / 100 \mathrm{~g})$ of raw materials (on dry weight basis).

\begin{tabular}{|l|c|}
\hline \multicolumn{1}{|c|}{ Samples } & Folic acid \\
\hline Whole wheat flour & 43 \\
\hline Wheat flour $(82 \%$ ext $)$ & 32 \\
\hline Wheat flour $(72 \%$ ext $)$ & 28 \\
\hline Fine bran & 316 \\
\hline Wheat germ meal & 286 \\
\hline Active dry yeast & 1652 \\
\hline
\end{tabular}

thus have significantly different folate contents. Fenech et al. (2005) found the aleurone together with germ and bran during milling . 


\subsection{Chemical composition of balady bread}

With regard to chemical composition of balady bread prepared from different formula (11 samples). The data in Table (4) showed statistically significant difference between the samples $(p \geq 0.05)$. The results in Table (4) indicated that replacement both wheat flour $(82$ and $72 \%$ extraction) by different levels of FB and WG led to augment the contents of protein (ranged from 10.25 to $16.68 \%$ ), fat (ranged from 1.15 to $4.54 \%$ ), fiber (ranged from 0.70 to $3.63 \%$ ) and ash (ranged from 0.98 to $2.87 \%$ ) in balady bread samples. These results are in agreement with those obtained by Sidhu et al.(1999) and Farouk et al. (2002) who found that addition of FB and WG to wheat flour led to increase the protein, fat, fiber and ash contents of bread.

\subsection{Folic acid content of balady bread}

In respct of TFA content of balady bread samples the present results (Fig. 1) elucidated that values of TFA content started with $124 \mu \mathrm{g} / 100 \mathrm{~g}$ in the sample No. (3) and reached to $318 \mu \mathrm{g} / 100 \mathrm{~g}$ in the sample No. (6). It is noteworthy from the same Figure that sample No. (4,6 \& 7) can supply about two - third TFA when comparing with Recommended Dietary Allowance (RDA) $(400 \mu \mathrm{g} / \mathrm{day})$ as mentioned by Stevenson et al. (2000). Thereafter, samples No. $(1,5,10$ \&11) can provide about half TFA when comparing with (RDA/d). Then samples No. (2,3,8 \&9) can give about third TFA when comparing with (RDA/d). The total folic acid loss between spiked flour and bread was investigated by some trials whereas Hefni and Witthöft (2011) evinced that baking process caused about 15\% TFA losses in baked bread. Also, Osseyi et al. (2001) and Kariluoto et al.(2004) evidenced that TFA losses due to baking process were about $20-25 \%$. With these values it can be postulated that FB and WG can be considered as a substantial sources of folic acid and as an alternative important strategy for increasing folic acid in the diet. Kariluoto et al. (2004) found that folate content increased during sourdough fermentation commonly used in rye baking and the increase was associated mainly with the growth of yeast. Yeast was able to compensate folate losses both in rye and wheat baking not only by its high folate content itself but also by synthesizing folates. There was no difference between total folate contents of wheat breads baked using either sponge-dough or straight- dough method. Changes in folate content during rye baking were accompanied by changes in vitamen distribution. Sourdough fermentation affects not only sensory and microbiological but also nutritional quality. The results suggest that by the means of screening and selecting appropriate yeast and lactic acid bacteria it could be possible to enhance natural folate content in bread. And so, Jägerstad et al. (2005) stated that some food process techniques can enhance folate content, e.g. bioprocessing including fermentation. Bread is sample of foods produced by the fermentation of yeast, itself a rich source of folate. Wheat breads baked using a sponge-dough or straight-dough method contained 2.5 times more folates than bread leavened with baking powder.

\subsection{Dietary fiber}

Respecting total dietary fiber (TDF) content in balady bread samples, the finding results in Table (5) demonstrated that sample No. (6) had the highest significant difference (14.53\%) in TDF content. On the contrary, sample No. (3) had the lowest significant difference $(5.25 \%)$ in TDF content. These results are in accordance with those mentioned by Sidhu et al. (1999) who found that TDF content in bread fortified by FB and WG was found to be far superior than that of the bread unfortified.

\subsection{Mineral contents}

Regarding mineral contents in balady bread samples the results in Table (5) elucidated that sample No. (7) had a maximum values of $\mathrm{Fe}, \mathrm{Zn}$ and $\mathrm{Ca}$ contents $(4.43,3.57 \& 49.91 \mathrm{mg} / 100 \mathrm{~g})$, respectively. In contrast, sample No. (3) had a minimum values of $\mathrm{Fe}, \mathrm{Zn}$ and $\mathrm{Ca}$ contents (2.42, $1.80 \& 45.16 \mathrm{mg} / 100 \mathrm{~g}$ ), respectively. These results are in concide with those obtained by Bedeir and Al Amri (2008) who proved that adding wheat germ to wheat flour led to ameliorate $\mathrm{Fe}, \mathrm{Zn}$ and $\mathrm{Ca}$ contents in bread supplemented with wheat germ compared to unsupplemented bread.

\subsection{Bread texture profile analysis}

Concerning bread texture profile analysis the results in Table (6) evident that the samples which recorded low values were more freshly (low staling rate), vice versa, samples which recorded high values were less freshly (high staling rate). The sample No. 4 was optimal and fresher than other samples in relation to firmness. This might be due to the high water absorption capacity of bran, consequently increase shelf-life of bread sample and enhance firmness of bread as mentioned by Laurikainen et al.(1998).

\subsection{Sensory evaluation}

In relation to sensory evaluation of balady bread prepared from different formulas, the results in Table (7) indicated that samples No. $(1,2,3$, $5,6,9,10$ and 11) were the best samples and were not significantly different for color and 
Production and evaluation of high folic acid

Table (4): Chemical composition (\%) of balady bread (on dry weight basis) *

\begin{tabular}{|c|c|c|c|c|c|}
\hline Samples No. & Protein & Fat & Fiber & Ash & Carbohydrates \\
\hline 1 & $15.34^{\mathrm{c}} \pm 0.012$ & $2.15^{\mathrm{f}} \pm 0.058$ & $3.11^{\mathrm{b}} \pm 0.015$ & $2.26^{\mathrm{b}} \pm 0.011$ & $77.14^{\mathrm{c}} \pm 0.237$ \\
\hline 2 & $11.79^{\mathrm{h}} \pm 0.01$ & $1.82^{\mathrm{g}} \pm 0.012$ & $1.94^{\mathrm{f}} \pm 0.023$ & $2.03^{\mathrm{g}} \pm 0.008$ & $82.42^{\mathrm{b}} \pm 0.242$ \\
\hline 3 & $10.25^{\mathrm{i}} \pm 0.028$ & $1.15^{\mathrm{h}} \pm 0.057$ & $0.70^{\mathrm{g}} \pm 0.012$ & $0.98^{\mathrm{i}} \pm 0.011$ & $86.92^{\mathrm{a}} \pm 0.294$ \\
\hline 4 & $15.54^{\mathrm{c}} \pm 0.063$ & $2.54^{\mathrm{e}} \pm 0.145$ & $3.63^{\mathrm{a}} \pm 0.008$ & $2.87^{\mathrm{a}} \pm 0.017$ & $75.42^{\mathrm{g}} \pm 0.173$ \\
\hline 5 & $15.60^{\mathrm{c}} \pm 0.057$ & $4.54^{\mathrm{a}} \pm 0.034$ & $2.56^{\mathrm{b}} \pm 0.021$ & $2.48^{\mathrm{d}} \pm 0.017$ & $74.82^{\mathrm{g}} \pm 0.232$ \\
\hline 6 & $15.93^{\mathrm{b}} \pm 0.011$ & $3.49^{\mathrm{c}} \pm 0.005$ & $3.50^{\mathrm{a}} \pm 0.577$ & $2.69^{\mathrm{c}} \pm 0.017$ & $74.39^{\mathrm{h}} \pm 0.173$ \\
\hline 7 & $16.68^{\mathrm{a}} \pm 0.14$ & $4.45^{\mathrm{a}} \pm 0.029$ & $3.22^{\mathrm{b}} \pm 0.115$ & $2.57^{\mathrm{d}} \pm 0.067$ & $73.08^{\mathrm{i}} \pm 0.046$ \\
\hline 8 & $12.60^{\mathrm{g}} \pm 0.11$ & $2.00^{\mathrm{f}} \pm 0.115$ & $2.98^{\mathrm{c}} \pm 0.017$ & $1.84^{\mathrm{h}} \pm 0.015$ & $80.58^{\mathrm{c}} \pm 0.288$ \\
\hline 9 & $13.23^{\mathrm{f}} \pm 0.11$ & $4.29^{\mathrm{a}} \pm 0.059$ & $2.13^{\mathrm{e}} \pm 0.057$ & $2.39^{\mathrm{f}} \pm 0.006$ & $77.96^{\mathrm{d}} \pm 0.248$ \\
\hline 10 & $14.06^{\mathrm{e}} \pm 0.24$ & $3.24^{\mathrm{d}} \pm 0.063$ & $2.54^{\mathrm{d}} \pm 0.083$ & $2.60^{\mathrm{c}} \pm 0.057$ & $77.56^{\mathrm{d}} \pm 0.323$ \\
\hline 11 & $14.78^{\mathrm{d}} \pm 0.034$ & $3.55^{\mathrm{c}} \pm 0.088$ & $2.45^{\mathrm{d}} \pm 0.021$ & $2.51^{\mathrm{d}} \pm 0.006$ & $76.71^{\mathrm{c}} \pm 0.346$ \\
\hline
\end{tabular}

*Values are mean $\pm \mathrm{SE}$

Table (5): TDF (\%) and mineral contents (mg/100 g) of balady bread (on dry weight basis)*.

\begin{tabular}{|c|c|c|c|c|}
\hline $\begin{array}{c}\text { Samples } \\
\text { No. }\end{array}$ & TDF & Fe & Zn & \multicolumn{1}{|c|}{ Ca } \\
\hline 1 & $13.89^{\mathrm{b}} \pm 0.031$ & $4.29^{\mathrm{a}} \pm 0.015$ & $3.45^{\mathrm{a}} \pm 0.008$ & $49.32^{\mathrm{a}} \pm 0.035$ \\
\hline 2 & $11.63^{\mathrm{d}} \pm 0.081$ & $3.47^{\mathrm{c}} \pm 0.028$ & $2.33^{\mathrm{d}} \pm 0.011$ & $46.35^{\mathrm{ab}} \pm 0.069$ \\
\hline 3 & $5.25^{\mathrm{g}} \pm 0.144$ & $2.42^{\mathrm{e}} \pm 0.018$ & $1.80^{\mathrm{e}} \pm 0.081$ & $45.61^{\mathrm{b}} \pm 0.080$ \\
\hline 4 & $13.73^{\mathrm{b}} \pm 0.112$ & $3.74^{\mathrm{b}} \pm 0.026$ & $2.41^{\mathrm{d}} \pm 0.031$ & $47.37^{\mathrm{ab}} \pm 0.298$ \\
\hline 5 & $11.91^{\mathrm{d}} \pm 0.153$ & $4.27^{\mathrm{a}} \pm 0.089$ & $3.42^{\mathrm{b}} \pm 0.088$ & $49.75^{\mathrm{a}} \pm 0.229$ \\
\hline 6 & $14.53^{\mathrm{a}} \pm 0.081$ & $4.36^{\mathrm{a}} \pm 0.043$ & $3.50^{\mathrm{a}} \pm 0.003$ & $49.86^{\mathrm{a}} \pm 0.199$ \\
\hline 7 & $12.61^{\mathrm{c}} \pm 0.057$ & $4.43^{\mathrm{a}} \pm 0.02$ & $3.57^{\mathrm{a}} \pm 0.010$ & $49.91^{\mathrm{a}} \pm 0.02$ \\
\hline 8 & $9.54^{\mathrm{e}} \pm 0.032$ & $2.92^{\mathrm{d}} \pm 0.003$ & $1.96^{\mathrm{e}} \pm 0.026$ & $46.40^{\mathrm{ab}} \pm 0.476$ \\
\hline 9 & $8.21^{\mathrm{f}} \pm 0.089$ & $3.30^{\mathrm{c}} \pm 0.014$ & $2.82^{\mathrm{c}} \pm 0.018$ & $47.25^{\mathrm{ab}} \pm 0.069$ \\
\hline 10 & $13.15^{\mathrm{c}} \pm 0.112$ & $3.51^{\mathrm{bc}} \pm 0.027$ & $2.84^{\mathrm{c}} \pm 0.011$ & $48.11^{\mathrm{ab}} \pm 0.041$ \\
\hline 11 & $11.36^{\mathrm{d}} \pm 0.064$ & $3.58^{\mathrm{b}} \pm 0.011$ & $2.96^{\mathrm{c}} \pm 0.015$ & $48.55^{\mathrm{ab}} \pm 0.109$ \\
\hline
\end{tabular}

*Values are mean $\pm \mathrm{SE}$

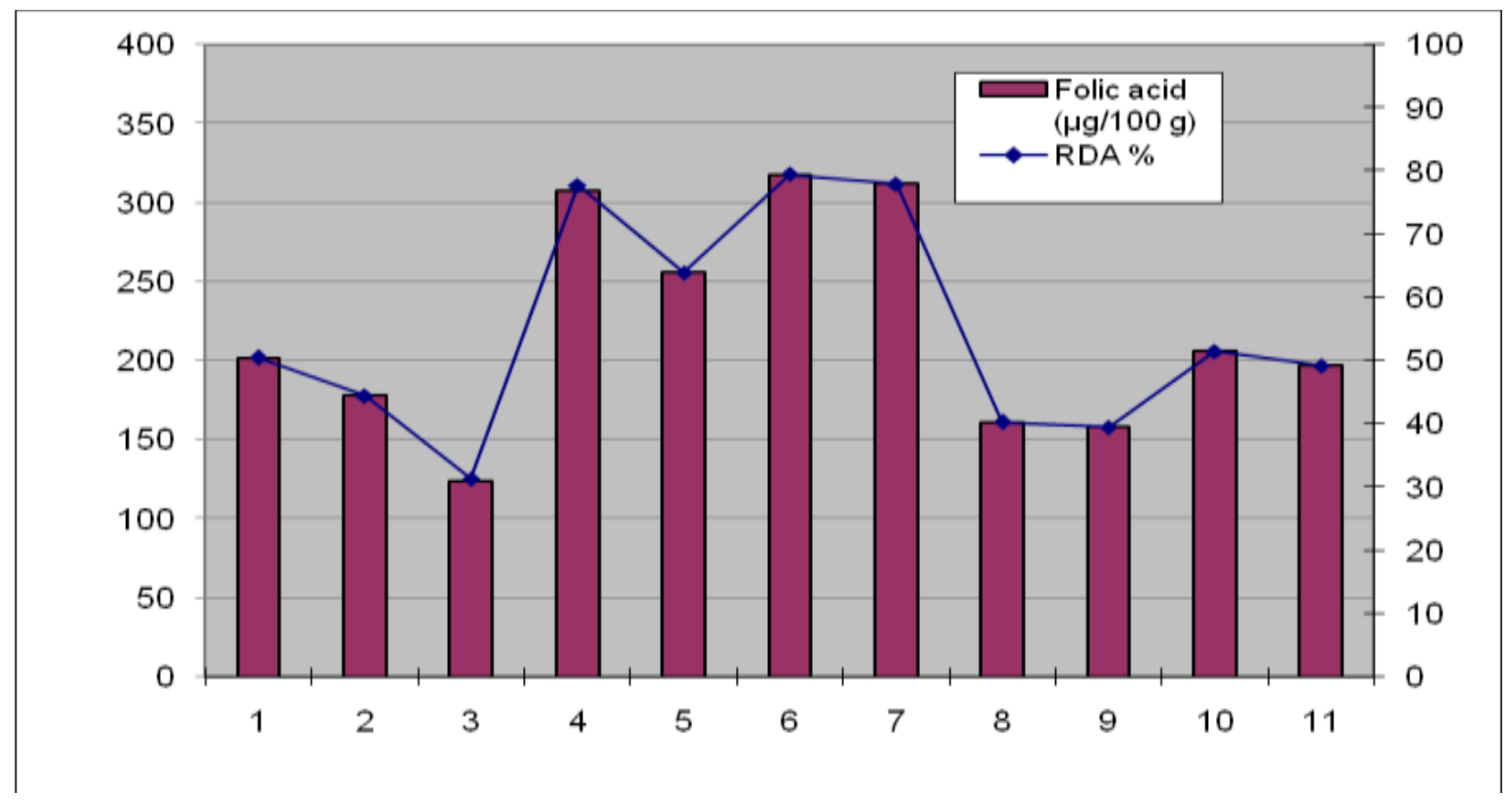

Fig. (1): Total folic acid content ( $\mu \mathrm{g} / 100 \mathrm{~g})$ and RDA (\%) of balady bread 
Table (6): Bread texture profile analysis.

\begin{tabular}{|c|c|c|c|c|c|c|}
\hline Samples No. & Firmness & Cohesiveness & Gumminess & Chewiness & Springiness & Resilience \\
\hline \multicolumn{7}{|c|}{ Bread texture profile analysis at zero time } \\
\hline 1 & 19.3 & 0.658098784 & 12.701307 & 8.90568 & 0.7011628 & 0.3883721 \\
\hline 2 & 16.59 & 0.783585487 & 12.999683 & 10.9005 & 0.8385236 & 0.6020761 \\
\hline 3 & 12.29 & 0.872295818 & 24.677249 & 21.5646 & 0.8738636 & 0.5034091 \\
\hline 4 & 23.2 & 0.83979226 & 19.48318 & 16.1146 & 0.8271028 & 0.5280374 \\
\hline 5 & 4.85 & 0.756577031 & 3.6693986 & 3.10987 & 0.8475138 & 0.5801105 \\
\hline 6 & 11.89 & 0.841809025 & 10.00911 & 8.8183 & 0.881024 & 0.487952 \\
\hline 7 & 9.51 & 0837202708 & 7.961798 & 7.1529 & 0.898409 & 0.634027 \\
\hline 8 & 9.65 & 0.85559749 & 8.2565158 & 6.74267 & 0.8166479 & 0.640045 \\
\hline 9 & 10.85 & 0.884156318 & 22.855441 & 18.6999 & 0.8181818 & 0.4306818 \\
\hline 10 & 5.6 & 1.226155444 & 3.8623896 & 3.62858 & 0.9394645 & 0.5296857 \\
\hline 11 & 4.63 & 0.67149013 & 1.839883 & 0.83065 & 0.4514673 & 0.5428894 \\
\hline \multicolumn{7}{|c|}{ Changes in bread texture profile analysis after 48 hours storage period. } \\
\hline 1 & 1.59 & 0.813 & 1.29 & 1.13 & 0.87 & 1.17 \\
\hline 2 & 2.75 & 0.672 & 1.85 & 1.44 & 0.78 & 0.67 \\
\hline 3 & 3.42 & 0.637 & 0.948 & 0.692 & $0.729 *$ & 0.787 \\
\hline 4 & $1.49 *$ & 0.856 & 1.53 & 1.467 & 0.954 & 1.09 \\
\hline 5 & $5.08 * *$ & $0.875^{* *}$ & 4.44 & 3.73 & 0.840 & 0.648 \\
\hline 6 & 2.73 & 0.863 & 2.36 & 1.96 & 0.830 & 1.06 \\
\hline 7 & 3.52 & 0.771 & 2.72 & 2.17 & 0.796 & $0.63 *$ \\
\hline 8 & 3.62 & 0.811 & 2.938 & 2.65 & 0.904 & 0.801 \\
\hline 9 & 2.31 & 0.627 & $0.608 *$ & $0.533^{*}$ & 0.876 & $1.20 * *$ \\
\hline 10 & 4.25 & $0.511^{*}$ & 3.87 & 2.97 & 0.769 & 0.76 \\
\hline 11 & 3.81 & 0.840 & $5.41^{* *}$ & $8.68 * *$ & $1.60 * *$ & 0.848 \\
\hline
\end{tabular}

* Low value.

** High value.

Table (7): Sensory evaluation of balady bread*

\begin{tabular}{|c|c|c|c|c|c|c|c|c|}
\hline No. & $\begin{array}{c}\text { Color \& } \\
\text { appearan } \\
\text { ce }(5)\end{array}$ & $\begin{array}{c}\text { Diameter } \\
\text { (5) }\end{array}$ & $\begin{array}{c}\text { Separation } \\
\text { (5) }\end{array}$ & $\begin{array}{c}\text { Biting } \\
\text { textures } \\
(5)\end{array}$ & $\begin{array}{c}\text { Aroma } \\
\text { (5) }\end{array}$ & $\begin{array}{c}\text { Taste } \\
\text { (5) }\end{array}$ & $\begin{array}{l}\text { Crumb } \\
\text { texture (5) }\end{array}$ & $\begin{array}{c}\text { Overall } \\
\text { score } \\
(35)\end{array}$ \\
\hline 1 & $\begin{array}{l}4.20^{\mathrm{ab}} \\
\pm 0.53\end{array}$ & $\begin{array}{c}4.25^{\mathrm{a}} \\
\pm 0.58\end{array}$ & $\begin{array}{c}4.30^{\mathrm{a}} \\
\pm 0.53\end{array}$ & $\begin{array}{l}3.80^{\mathrm{bc}} \\
\pm 0.58\end{array}$ & $\begin{array}{l}3.90^{\text {bcd }} \\
\pm 0.56\end{array}$ & $\begin{array}{l}4.00^{\mathrm{abc}} \\
\pm 0.70\end{array}$ & $\begin{array}{c}3.80^{\mathrm{a}} \\
\pm 0.53\end{array}$ & $\begin{array}{c}28.25^{\mathrm{abc}} \\
\pm 3.37\end{array}$ \\
\hline 2 & $\begin{array}{c}4.55^{\mathrm{a}} \\
\pm 0.43\end{array}$ & $\begin{array}{c}4.60^{\mathrm{a}} \\
\pm 0.31\end{array}$ & $\begin{array}{l}4.40^{\mathrm{a}} \\
\pm 0.51\end{array}$ & $\begin{array}{c}4.25^{\mathrm{a}} \\
\pm 0.26\end{array}$ & $\begin{array}{l}4.15^{\text {abc }} \\
\pm 0.24\end{array}$ & $\begin{array}{c}4.35^{\mathrm{a}} \\
\pm 0.24\end{array}$ & $\begin{array}{c}4.00^{\mathrm{a}} \\
\pm 0.23\end{array}$ & $\begin{array}{l}30.30^{\mathrm{a}} \\
\pm 1.43\end{array}$ \\
\hline 3 & $\begin{array}{l}4.55^{\mathrm{a}} \\
\pm 0.64\end{array}$ & $\begin{array}{l}4.60^{\mathrm{a}} \\
\pm 0.51\end{array}$ & $\begin{array}{l}4.55^{\mathrm{a}} \\
\pm 0.55\end{array}$ & $\begin{array}{l}4.15^{\mathrm{ab}} \\
\pm 0.57\end{array}$ & $\begin{array}{l}4.15^{\text {abc }} \\
\pm 0.47\end{array}$ & $\begin{array}{l}4.20^{\mathrm{ab}} \\
\pm 0.34 \\
\end{array}$ & $\begin{array}{l}4.00^{\mathrm{a}} \\
\pm 0.47\end{array}$ & $\begin{array}{l}30.20^{\mathrm{ab}} \\
\pm 3.17\end{array}$ \\
\hline 4 & $\begin{array}{l}3.70^{\mathrm{bc}} \\
\pm 0.67\end{array}$ & $\begin{array}{c}4.35^{\mathrm{a}} \\
\pm 0.70\end{array}$ & $\begin{array}{c}4.30^{\mathrm{a}} \\
\pm 0.67\end{array}$ & $\begin{array}{c}3.70^{\mathrm{c}} \\
\pm 0.42\end{array}$ & $\begin{array}{c}3.70^{\mathrm{d}} \\
\pm 0.67\end{array}$ & $\begin{array}{c}3.65^{\mathrm{c}} \\
\pm 0.62\end{array}$ & $\begin{array}{c}3.35^{\mathrm{b}} \\
\pm 0.62\end{array}$ & $\begin{array}{l}26.75^{\mathrm{c}} \\
\pm 3.18\end{array}$ \\
\hline 5 & $\begin{array}{l}4.20^{\mathrm{ab}} \\
\pm 0.34\end{array}$ & $\begin{array}{r}4.25^{\mathrm{a}} \\
\pm 0.58\end{array}$ & $\begin{array}{r}4.25^{\mathrm{a}} \\
\pm 0.63\end{array}$ & $\begin{array}{l}3.90^{\text {abc }} \\
\pm 0.31\end{array}$ & $\begin{array}{l}3.85^{\mathrm{cd}} \\
\pm 0.33\end{array}$ & $\begin{array}{l}3.90^{\mathrm{bc}} \\
\pm 0.45\end{array}$ & $\begin{array}{l}3.65^{\mathrm{ab}} \\
\pm 0.52\end{array}$ & $\begin{array}{l}28.00^{\mathrm{bc}} \\
\pm 2.19\end{array}$ \\
\hline 6 & $\begin{array}{l}3.95^{\mathrm{b}} \\
\pm 0.36\end{array}$ & $\begin{array}{c}4.55^{\mathrm{a}} \\
\pm 0.43\end{array}$ & $\begin{array}{c}4.55^{\mathrm{a}} \\
\pm 0.43\end{array}$ & $\begin{array}{l}3.85^{\text {abc }} \\
\pm 0.33\end{array}$ & $\begin{array}{l}4.25^{\text {abc }} \\
\pm 0.26\end{array}$ & $\begin{array}{l}4.20^{\mathrm{ab}} \\
\pm 0.25\end{array}$ & $\begin{array}{l}3.65^{\text {ab }} \\
\pm 0.41\end{array}$ & $\begin{array}{c}29.00^{\mathrm{ab}} \\
\pm 1.20\end{array}$ \\
\hline 7 & $\begin{array}{l}3.95^{\mathrm{b}} \\
\pm 0.43\end{array}$ & $\begin{array}{c}4.45^{\mathrm{a}} \\
\pm 0.49\end{array}$ & $\begin{array}{r}4.45^{\mathrm{a}} \\
\pm 0.49\end{array}$ & $\begin{array}{l}3.80^{\mathrm{bc}} \\
\pm 0.48\end{array}$ & $\begin{array}{l}4.30^{\mathrm{ab}} \\
\pm 0.25\end{array}$ & $\begin{array}{l}4.10^{\mathrm{ab}} \\
\pm 0.21\end{array}$ & $\begin{array}{c}3.90^{\mathrm{a}} \\
\pm 0.31\end{array}$ & $\begin{array}{c}28.95^{\text {ab }} \\
\pm 1.72\end{array}$ \\
\hline 8 & $\begin{array}{l}4.40^{\mathrm{ab}} \\
\pm 0.21\end{array}$ & $\begin{array}{l}4.50^{\mathrm{a}} \\
\pm 0.40\end{array}$ & $\begin{array}{l}4.60^{\mathrm{a}} \\
\pm 0.39\end{array}$ & $\begin{array}{l}4.25^{\mathrm{a}} \\
\pm 0.54\end{array}$ & $\begin{array}{c}4.40^{\mathrm{a}} \\
\pm 0.39\end{array}$ & $\begin{array}{l}4.25^{\mathrm{ab}} \\
\pm 0.26\end{array}$ & $\begin{array}{c}4.05^{\mathrm{a}} \\
\pm 0.43\end{array}$ & $\begin{array}{l}30.45^{\mathrm{a}} \\
\pm 1.36\end{array}$ \\
\hline 9 & $\begin{array}{l}4.25^{\mathrm{ab}} \\
\pm 0.35\end{array}$ & $\begin{array}{l}4.40^{\mathrm{a}} \\
\pm 0.51\end{array}$ & $\begin{array}{l}4.25^{\mathrm{a}} \\
\pm 0.48\end{array}$ & $\begin{array}{l}4.05^{\mathrm{abc}} \\
\pm 0.15\end{array}$ & $\begin{array}{c}4.00^{\text {abcd }} \\
\pm 0.23\end{array}$ & $\begin{array}{l}3.90^{\mathrm{bc}} \\
\pm 0.39\end{array}$ & $\begin{array}{c}3.85^{\mathrm{a}} \\
\pm 0.47\end{array}$ & $\begin{array}{c}28.70^{\mathrm{abc}} \\
\pm 1.53\end{array}$ \\
\hline 10 & $\begin{array}{l}4.20^{\mathrm{ab}} \\
\pm 0.25\end{array}$ & $\begin{array}{l}4.50^{\mathrm{a}} \\
\pm 0.47\end{array}$ & $\begin{array}{c}4.50^{\mathrm{a}} \\
\pm 0.47\end{array}$ & $\begin{array}{l}3.95^{\mathrm{abc}} \\
\pm 0.36\end{array}$ & $\begin{array}{l}4.30^{\mathrm{ab}} \\
\pm 0.42\end{array}$ & $\begin{array}{l}4.15^{\mathrm{ab}} \\
\pm 0.33\end{array}$ & $\begin{array}{c}3.85^{\mathrm{a}} \\
\pm 0.33\end{array}$ & $\begin{array}{c}29.45^{\text {ab }} \\
\pm 1.73\end{array}$ \\
\hline 11 & $\begin{array}{l}4.20^{\mathrm{ab}} \\
\pm 0.25\end{array}$ & $\begin{array}{c}4.50^{\mathrm{a}} \\
\pm 0.40\end{array}$ & $\begin{array}{l}4.45^{\mathrm{a}} \\
\pm 0.43\end{array}$ & $\begin{array}{l}3.95^{\text {abc }} \\
\pm 0.15\end{array}$ & $\begin{array}{l}4.25^{\text {abc }} \\
\pm 0.42\end{array}$ & $\begin{array}{l}4.10^{\mathrm{ab}} \\
\pm 0.31\end{array}$ & $\begin{array}{c}3.80^{\mathrm{a}} \\
\pm 0.25 \\
\end{array}$ & $\begin{array}{c}29.25^{\mathrm{ab}} \\
\pm 1.35\end{array}$ \\
\hline
\end{tabular}

*Values followed by the same letter within the same column were not significantly different at $p \geq 0.05$.

* Values are mean \pm standard deviation. 
appearance compared to other samples. Another samples showed adverse effect on color and appearance as a result of incorporation of different levels of FB and WG. From the same table it is obvious that there were no significant differences among all samples for diameter and layers separation. Also, the same results appeared that samples No. (2, 3, 5, 6, 9, 10 and 11) were the best and were not significantly different for biting textures compared to other samples. Furthermore, samples No. (2, 3, 6, 7, 9, 10 and 11) the best and were not significantly different for aroma compared to other samples. Besides, samples No. $(1,2,3,6,7,8,10$ and 11) were the best and were not significantly different for taste compared to other samples. Moreover, samples No. (1, 2, 3, 5, $6,7,8,9,10 \& 11)$ were the best and were not significantly different for crumb texture compared to sample No. 4. These results are agreed with those obtained by $\mathrm{Li}$ et al.(1997) who found improvement in crumb texture in pan bread fortified with wheat germ. This could be imputed to wheat germ possess high fat content. On the other side, Salmenkallio - Marttila et al. (2001) proved that wheat bran can decrease the volume bread, elasticity and weakens the structure. This perhaps attributed to that bran had high fiber content which destroy the gluten matrix. Therewithal samples No. (1, 2, 3, 6, 7, 8,9, 10 and 11) recorded higher overall score and were not significant than that of other samples.

It can be concluded that folic acid contents increased in balady bread baked with either fine bran or wheat germ. They could be used as a partial replacement for wheat flour in bread baking. A balady bread bio-fortified with folic acid that is acceptable to Egyptian consumer could be produced. In view of balady bread in Egypt,it is considered as backbone in diets, therefore, the aforementioned results deduced that there is an urgent need for increase concentration, the light focus on balady bread as a pattern for folic acid enrichment.

\section{REFERENCES}

AACC (2000). Approved methods of American association of cereal chemists $\left(10^{\text {th }}\right.$ ed.). St Paul: American Association of Cereal Chemists, Inc.

AOAC (2000). Official methods of analysis $\left(17^{\text {th }}\right.$ ed.). Association of Official Analytical Chemists. Arlington, Virginia, USA.

Bedeir S. H. and Al Amri M.S. (2008). Effect of adding milled defatted roasted wheat germ on chemical composition, nutritional value and quality of high fiber bread. Egyptian J. Nutr. 2: 157-176.

Egypt Demographic and Health Survey (EDHS). (2005). National population council. Cairo, Egypt.

FAO (2005) Food and Agriculture Organization. Food balance sheet.

<http://www.fao.org>Accessed 04.11.07.

Farouk A.G., Asad E.M. and El-Sayed S.M. (2002). Nutritional and rhealogical studies on some types of balady bread. J. Agric. Sci., Mansoura Univ., 27 (6): 3945-3952.

Fenech M., Noakes M., Clifton P. and Topping D. (1999). Aleurone flour is a rich source of bioavailable folate in humans. J. Nutr., 129: 1114-1119.

Fenech M., Noakes M., Clifton P. and Topping D. (2005). Aleurone flour increases red-cell folate and lowers plasma homocyst(e)ine substantially in man. Brit. J. Nutr., 93; 353360.

Hefni M., Öhrvik V., Tabekha M. and Witthöft C. (2010). Folate content in foods commonly consumed in Egypt. Food Chem., 121: 540545.

Hefni M. and Witthöft C. (2011). Increasing the folate content in Egyptian balady bread using germinated wheat flour. LWT- Food Science and Technology, 44 :706-712.

Hefni M. and Witthöft C. (2012). Effect of germination and subsequent oven - drying on folate content in different wheat and rye cultivars. J. Cereal Science, 56: 374-378.

Houghton L., Gray A., Rose M. Miller J.,Hurthouse N. and Gregory J. (2011). Longterm effect of low-dose folic acid intake: potential effect of mandatory fortification on the prevention of neural tube defects. Am. J. Clin. Nutr., 94: 136-141.

Jägerstad M., Pijronen V.,Walker C., Ros G., Carnovale E., Holasova M. and Nau H. (2005). Increasing natural food folates through bioprocessing and biotechnology. Trends in Food Science \& Technology, 16: 298-306.

Kariluoto S., Vahteristo L., Salovaara H., Katina K., Liukkonen K. and Piironen V. (2004). Effect of baking method and fermentation of folate content of rye and wheat breads. Cereal Chem., 81 (1): 134-139.

Katina K.,Liukkonen K., Kaukovirta - Norja, A., Adlercreutz, H., Heinonen S., Lampi A., Pihlava J. and Poutanen, K. (2007). Fermentation - induced changes in the nutritional value of native or germinated rye. J. Cereal Science, 46: 348-355. 
Kim J., Cho Y., Kim D., Lee B., Hwang D., Jeong J., Lee H., Matsuo K., Tajima K. and Ahn Y. (2012). Dietary intake of folate and alcohol, MHFR C677T polymorphism and colorectal cancer risk in Korea. Am. J. Clin. Nutr., 95: 405-412.

Laurikainen I., Härkönen H., Autro K. and Poutanen K. (1998). Effects of enzymes in fibre-enriched baking. J. Science Food and Agric., 70: 239-249.

Li Y., Chen N. and Zhao X. (1997). Effect of wheat germ and its products on the baking quality of flour. The Chinese Cereal and Oils Association, (1): 32-39.

Louz S.L., Hussein A.S. and Shabib Z.A. (2010). Development of balady bread supplemented with cumin and low-fat soybean flour. Egyptian J. Nutr., 3: 45-67.

Metwalli A.A. and Abdelrasoul E.A. (2010). Utilization of banana flour as nutrient supplement of wheat flour. Egyptian J. Nutr., 3: 101-127.

Mohamed M., Atia A., Hussein A. and Assem N. (1996). Effect of wheat flour and yeast on balady bread characteristics. Egypt. J. Food Sci., 24 (1): 81-92.

Osseyi E.S., Wehling R.L. and Albrecht J.A. (2001). HPLC determination of stability and distribution of added folic acid and some endogenous folates during bread making. Cereal Chem., 78 (4): 375-378.

Póo-Prieto R., Haytowitz D., Holden J., Rogers G., Choumenkovitch S., Jacques P. and Selhub J. (2006). Use of the affinity/HPLC method for quantitative estimation of folic acid in enriched cereal-grain products. J. Nutr.,136: 3079-3083.

Reed G. and Nagodawithenan T. (1991). Baker's yeast production. In: Yeast Technology. pp. 261-313 ( $2^{\text {nd }}$ ed). Van Nostrand Reinhold New York.

Salmenkallio-Marttila M., Katina K. and Autio K. (2001). Effects of bran fermentation on quality and microstructure of high-fibre wheat bread. Cereal Chem., 78: 429-435.

Sidhu J., Al-Hooti S. and Al-Saqer J. (1999). Effect of adding wheat bran and germ fractions on the chemical composition of high-fiber toast bread. Food Chem., 67 (4): 365-371.

Sourki A., Davoodi M.,Yazdi F.,Mortazavi S., Karimi M., Jahromi S. and Pourfarzad A. (2010). Staling and quality of Iranian flat bread stored at modified atmosphere in different packaging. World Academy of Science Engineering and Technology, 69: 390-395.

SPSS (1998). Statistical Algorithm, SPSS Inc., Reports Library of Congress, US.

Stevenson R., Allen W., Pai G., Best R., Seaver R., Dean J. and Thompson S. (2000). Decline in prevalence of neural tube defects in a highrisk region of the United States. Pediatrics. 106: 677-683.

Walker J. Batterham P., Mackinnon A., Jorm A., Hickie L., Fenech M., Kljakovic M., Crisp D. and Christensen H. (2012). Oral folic acid and vitamin B-12 supplementation to prevent cognitive decline in community - dwelling adults with depressive symptoms - the Beyond Ageing Project: a randomized controlled trial. Am. J. Clin. Nutr., 95: 194-203.

West A., Yan J., Perry C., Jiang X., Malysheva O. and Caudill M. (2012). Folate-status response to a controlled folate intake in nonpregnant, pregnant and lactating women. Am. J. Clin. Nutr., 96: 789-800. 


\section{إنتاج وتقييم الخبز البلدى عـالى المحتوى من حمض الفوليك \\ أيمن عزت محم - حنان فوزى أحمد- منال عبدالمطلع عطوه*- ناديه محمود عبدالمطلب}

معهد بحوث تكنولوجيا الأغذية ـ * المركز الإقليمى للأغذية والأعلاف

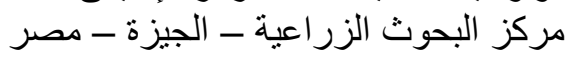

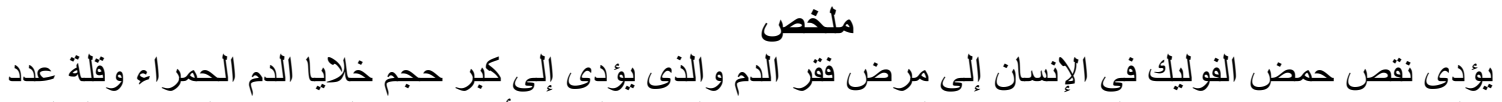

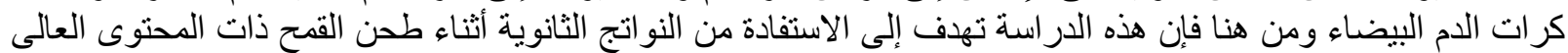

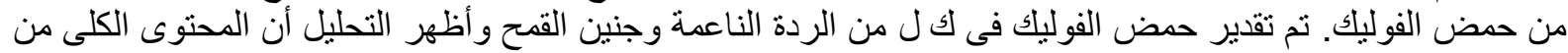

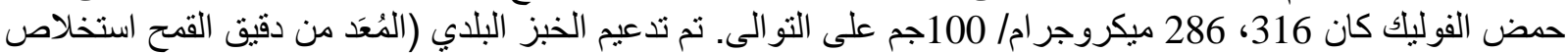

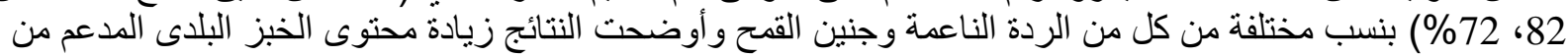

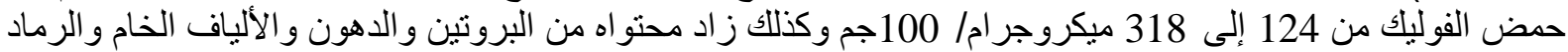

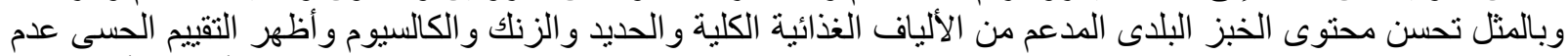

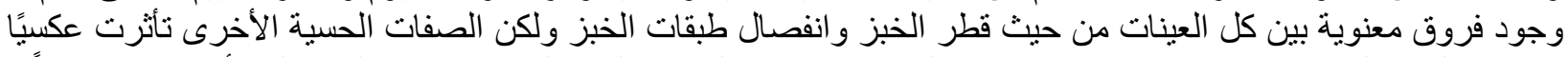

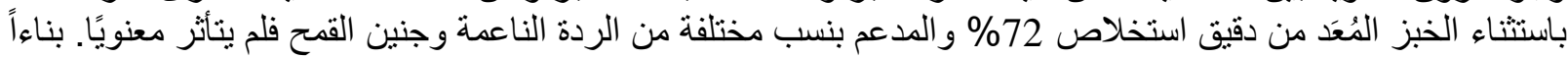

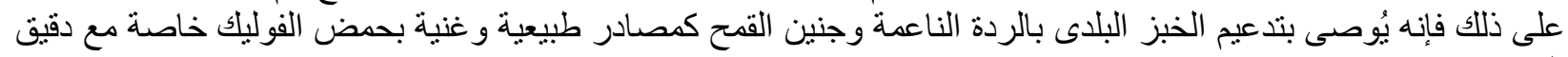

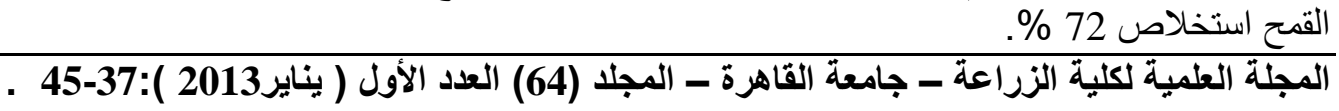

